\title{
Impact of common type 2 diabetes risk gene variants on future type 2 diabetes in the non-diabetic population in Korea
}

\author{
Se Eun Park ${ }^{1}$, Won Young Lee ${ }^{1}$, Ki Won $\mathrm{Oh}^{1}$, Ki Hyun Baek ${ }^{2}$, Kun Ho Yoon ${ }^{2}$, Moo Il Kang ${ }^{2}$, Ho Young Son ${ }^{2}$ \\ and Won Chul Lee ${ }^{3}$
}

We prospectively examined the association between type 2 diabetes mellitus (T2DM) progression and common T2DM-risk gene variants in $\mathbf{8 7 0}$ non-diabetic participants in a Chungju Metabolic Disease Cohort Study in Korea. We genotyped the following six single nucleotide polymorphisms (SNPs): KCNQ1 (potassium voltage-gated channel, KQT-like subfamily member 1) rs2237892, CDKAL1 (regulatory subunit-associated protein 1-like 1) rs7554840, CDKN2A/B (cyclin-dependent kinase inhibitor 2A/B) rs1081161, SCL3OA8 (solute carrier family 30 member 8 gene) rs13266634, TCF7L2 (transcription factor 7-like 2) rs7903146, and PPARG (peroxisome proliferator activated receptor gamma) rs1801282. Anthropometric data and metabolic parameters were obtained at baseline and year 4 . Pancreatic $\beta$ cell function was assessed by the homeostasis model assessment index of $\beta$ cells (HOMA- $\beta$ ). After 4 years, 137 subjects developed T2DM (15.7\%). A significant association was found in the variant of KCNQ1 rs2237892, whereas the SNPs of CDKAL1, CDKN2A/B, SCL3OA8, TCF7L2 and PPARG were not associated. The C-allele carriers of KCNQ1 conferred a significantly increased risk for T2DM compared with the T/T genotype, independently of clinical risk factors (odds ratio $=2.61,95 \%$ confidence intervals $=1.02-6.69, P=0.04$ ). Although no differences were observed at baseline among the KCNQ1 variants, HOMA- $\beta$ levels by year 4 were significantly lower in the $\mathrm{C}$-allele carriers after controlling for metabolic parameters. The genetic variations in KCNQ1 are associated with future development of T2DM in Koreans, which might be mediated by differences in insulin secretory function.

Journal of Human Genetics (2012) 57, 265-268; doi:10.1038/jhg.2012.16; published online 1 March 2012

Keywords: genetic association; KCNQ1; type 2 diabetes mellitus

\section{INTRODUCTION}

Type 2 diabetes mellitus (T2DM) is a chronic metabolic disorder characterized by hyperglycemia, variable degrees of insulin resistance, impaired insulin secretion and increased hepatic glucose production. ${ }^{1}$ The prevalence of diabetes is increasing and expected to rise more rapidly in the future as obesity increases, the population becomes older and the physical activity levels of most people decrease. ${ }^{2,3}$

In addition to the environmental factors mentioned above, genetic components are obviously associated. Recent genome-wide association studies have identified several genetic variants that seem to increase the risk of T2DM. ${ }^{4-9}$ Some novel loci identified by genome-wide association studies were also found in the Asian population. ${ }^{10,11}$ Previous studies reported that some diabetes-risk gene variants were associated with the progression of T2DM. ${ }^{12,13}$ However, few data have been reported about the relationship between diabetessusceptibility loci and the progression of T2DM in non-diabetic participants, especially in the Asian population. In addition, it has recently been reported that there are significant differences in the contribution of known single nucleotide polymorphisms (SNPs) in susceptibility genes among various ethnic populations. ${ }^{5,14,15}$ Therefore, studies in different populations are also important.

The aim of this study was to investigate whether variations in recently identified diabetes-susceptibility genes-KCNQ1 (potassium voltagegated channel, KQT-like subfamily, member 1), regulatory subunitassociated protein 1-like 1 (CDKAL1), cyclin-dependent kinase inhibitor 2A/B (CDKN2A/B), solute carrier family 30 member 8 gene (SCL30A8), transcription factor 7-like 2 (TCF7L2) and peroxisome proliferator activated receptor gamma $(P P A R G)$ - could predict the development of T2DM in non-diabetic subjects from the Korean population.

\section{MATERIALS AND METHODS}

Participants

These analyses were based on information obtained from participants in the Chungju Metabolic Disease Cohort Study, which is an ongoing prospective

${ }^{1}$ Department of Endocrinology and Metabolism, Kangbuk Samsung Hospital, Sungkyunkwan University School of Medicine, Seoul, Korea; ${ }^{2}$ Department of Endocrinology and Metabolism, The Catholic University of Korea, College of Medicine, Seoul, Korea and ${ }^{3}$ Department of Preventive Medicine, The Catholic University of Korea, College of Medicine, Seoul, Korea

Correspondence: Professor KW Oh, Division of Endocrinology and Metabolism, Department of Internal Medicine, Kangbuk Samsung Hospital, Sungkyunkwan University School of Medicine, no. 108 Pyung-Dong, Jongro-Ku, Seoul 110-746, South Korea.

E-mail: okwendo@yahoo.co.kr

Received 16 August 2011; revised 3 January 2012; accepted 23 January 2012; published online 1 March 2012 
cohort study that began in 2003 in Korea. ${ }^{16}$ A total of 1337 individuals (509 men and 828 women) participated in this study from May 2007 to April 2008, enrolled by stratified random cluster sampling. The investigated group consisted of adults aged $>40$ years and living in a rural area near Chungju. Among the 1337 participants in 2007, 1172 subjects had been seen previously (from May 2003 to April 2004) and returned 4 years later for follow-up (from May 2007 to April 2008).

Patients were eligible to participate in the study if they had no previous diagnosis of diabetes and their fasting plasma glucose (FPG) level was $<7.0 \mathrm{mmoll}^{-1}$ in $2003 .{ }^{17}$ Patients were excluded if they had a history of diabetes or other severe metabolic disorders (for example, hyperthyroidism, hypothyroidism, Cushing's syndrome, acromegaly or pheochromocytoma), infectious disease or a recorded FPG level $>7.0 \mathrm{mmoll}^{-1}$. After our screening criteria were applied, a total of 870 subjects were selected for study. At year 4 , the development of diabetes was assessed from patient records or by a FPG $\geqslant 7.0 \mathrm{mmoll}^{-1}$.

Written informed consent was obtained from each participant, and the study protocol adhered to the ethical guidelines of the 1975 Declaration of Helsinki, as reflected in a priori approval by the institution's Human Research Committee.

\section{Anthropometric and laboratory measurements}

Anthropometric data and laboratory parameters were obtained at baseline and year 4. Height and weight measurements were taken in 2003 and 2007. The body mass index $\left(\mathrm{kg} \mathrm{m}^{-2}\right)$ was calculated as body weight in kilograms divided by the square of height in meters. Systolic and diastolic blood pressure was measured in duplicate, and the results were averaged.

Fasting blood samples were collected for the measurement of plasma glucose, total cholesterol, triglyceride, high-density lipoprotein cholesterol and low-density lipoprotein cholesterol. Blood analysis was performed at a central laboratory (Samkwang Medical Laboratories, Seoul, Korea) for both accuracy and consistency. Total cholesterol and triglyceride levels were measured using an enzymatic calorimetric test, high-density lipoprotein (cholesterol was measured using the selective inhibition method, and low-density lipoprotein (cholesterol was calculated with the Friedewald formula. FPG levels were measured using sodium fluoride tubes and analyzed using the hexokinase method. Fasting plasma insulin levels were measured using a radioimmunoassay kit (Dainabot, Tokyo, Japan). Insulin resistance was assessed using the homeostasis model assessment of insulin resistance according to the following equation: fasting plasma insulin $\left(\mathrm{uU} \mathrm{ml}^{-1}\right) \times$ fasting blood glucose $\left(\mathrm{mmoll}^{-1}\right)$ / 22.5. The homeostasis model assessment index of $\beta$ cells (HOMA- $\beta$ ) index is an approximation of insulin secretion, and this index was defined as (fasting plasma insulin $\left.\left(\mu \mathrm{U} \mathrm{ml}^{-1}\right) \times 20\right) /\left(\mathrm{FPG}\left(\mathrm{mmoll}^{-1}\right)-3.5\right) .{ }^{18}$

\section{Gene and SNP selection}

SNPs from six novel genetic loci, identified from the recent genome-wide association studies, were selected for this study. ${ }^{4,7,9-11}$ In particular, we selected six genes that showed reproducible associations with T2DM in cross-sectional studies in Korea. ${ }^{19-22}$ The selected genes were as follows: KCNQ1 rs2237892, CDKAL1 rs7554840, CDKN2A/B rs1081161, SCL30A8 rs13266634, TCF7L2 rs7903146 and PPARG rs1801282.

\section{DNA extraction and genotyping}

The buffy coat was separated from blood samples of the study subjects and stored at $70^{\circ} \mathrm{C}$, and genomic DNA was extracted using a DNA purification kit (Takara Bio Inc., Otsu, Japan). The genotyping of the six polymorphisms in the KCNQ1, CDKAL1, CDKN2A/B, SCL30A8, TCF7L2 and PPARG genes was performed by allelic discrimination using the $5^{\prime}$-nuclease PCR assay. The detector used in this experiment was an ABI Prism 7200 sequence detection platform (Perkin Elmer, Branford, CT, USA). The primers and probes used for genotyping analyses are shown in Supplementary Table S1.

\section{Statistical analyses}

The genotype frequencies were tested for Hardy-Weinberg equilibrium using the $\chi^{2}$ test. We used the analysis of variance, Wilcoxon Rank-sum test and Kruskal-Wallis test for comparison of continuous variables and the $\chi^{2}$ test for comparison of categorical variables. To adjust for metabolic parameters, multivariate logistic regression tests were used, and odds ratios and 95\% confidence intervals were calculated for the development of T2DM after 4 years. For these calculations, patients with the non-risk allele were denoted as zero, heterozygotes were denoted as one and patients who were homozygous for the risk allele were denoted as two. Analysis of covariance was used to compare the variables, controlling for metabolic parameters. All analyses were performed using SPSS for Windows software version 17.0 (SPSS, Chicago, IL, USA). Power calculations were performed using PASS software version 2008 (NCSS Statistical Software, Kaysville, UT, USA). The estimated powers of our study were $97 \%, 72 \%, 98 \%$ and $39 \%$ for rs2237892 (KCNQ1), rs7754840 (CDKAL1), rs10811661 (CDKN2A/B) and rs13266634 (SLC30A8), respectively, in the recessive genetic model and $92 \%, 84 \%, 85 \%, 61 \%, 85 \%$ and $41 \%$ for rs2237892 (KCNQ1), rs7754840 (CDKAL1), rs10811661 (CDKN2A/B), rs13266634 (SLC30A8), rs7903146 (TCF7L2) and rs1801282 (PPARG), respectively, in the dominant genetic model, based on the reference odds ratios and allele frequencies from previous studies. ${ }^{15,21,23} P<0.05$ was considered statistically significant.

\section{RESULTS}

Genotyping of six representative SNPs from T2DM susceptibility genes were replicated in 870 non-diabetic subjects from the Korean population. The baseline clinical and biochemical characteristics of the subjects are shown in Table 1 . The genotype and allele frequencies of T2DM susceptibility gene SNPs are presented in Table 2. All genotype distributions were in agreement with Hardy-Weinberg equilibrium. The SCL30A8 rs13266634, CDKN2A/B rs1081161, KCNQ1 rs2237892 and PPARG rs1801282 risk alleles were considered major alleles, whereas the other two risk alleles were minor alleles in our population, consistent with previous reports in European populations. ${ }^{6,7,9}$

No significant differences were seen in baseline FPG levels for the risk alleles (Supplementary Table S2). Of the 870 non-diabetic subjects investigated at baseline, 137 subjects (15.7\%) developed T2DM after 4 years.

For each SNP shown to be a possible T2DM-susceptible variant according to our study, we further analyzed the effect of genotypes under the two different genetic models (Table 3). After adjustment for several clinical risk factors, KCNQ1 rs2237892 was significantly associated with the progression of T2DM after 4 years in the additive model $(P=0.04)$. The $\mathrm{C} / \mathrm{C}$ and $\mathrm{C} / \mathrm{T}$ genotypes of KCNQ1 conferred a significantly increased risk for T2DM compared with the $\mathrm{T} / \mathrm{T}$

\section{Table 1 Clinical characteristics of the study population}

\begin{tabular}{lc}
\hline Variable & Mean \pm s.d. \\
\hline Age (years) & $61.58 \pm 9.31$ \\
Sex, M/F & $393 / 477$ \\
BMI (kg m ${ }^{-2}$ ) & $24.04 \pm 3.36$ \\
Waist circumference (cm) & $85.75 \pm 8.38$ \\
Systolic blood pressure (mm Hg) & $139.87 \pm 21.97$ \\
Diastolic blood pressure (mm Hg) & $86.27 \pm 11.63$ \\
FBG (mmol I-1) & $5.23 \pm 0.60$ \\
Total cholesterol (mmol I-1) & $5.16 \pm 0.99$ \\
Triglyceride (mmol I-1) & $1.45(1.03-2.17)$ \\
HDL-C (mmol I-1) & $1.25 \pm 0.21$ \\
LDL-C (mmol I ${ }^{-1}$ ) & $3.13 \pm 0.88$ \\
Fasting insulin (pmol I-1) & $30.56(19.45-50.18)$ \\
HOMA-IR (arbitrary unit) & $1.04(0.60-1.68)$ \\
HOMA- $\beta$ (arbitrary unit) & $55.03(36.13-84.77)$
\end{tabular}

Abbreviations: BMI, body mass index; FBG, fasting blood glucose; HDL-C, high-density lipoprotein cholesterol; HOMA- $\beta$, homeostasis model assessment of $\beta$ cell function; HOMA-IR, homeostasis model assessment of insulin resistance; LDL-C, low-density lipoprotein cholesterol. The parameters that did not follow the normal distribution were presented as the median and interquartile range. 
Table 2 Genotype frequencies of candidate SNPs with T2DM

\begin{tabular}{lllll}
\hline Gene (rs number) & $\begin{array}{c}\text { Allele } \\
\text { (major/minor) }\end{array}$ & Genotype & Number & HWE (P) \\
\hline KCNQ1 (rs2237892) & $\mathrm{C} / \mathrm{T}$ & $\mathrm{CC}$ & 314 & 0.401 \\
& & $\mathrm{CT}$ & 433 & \\
& & $\mathrm{TT}$ & 123 & \\
CDKAL1 (rs7554840) & $\mathrm{G} / \mathrm{C}$ & $\mathrm{GG}$ & 245 & 0.610 \\
& & $\mathrm{GC}$ & 447 & \\
& & $\mathrm{CC}$ & 178 & \\
CDKN2A/B (rs1081161) & $\mathrm{T} / \mathrm{C}$ & $\mathrm{TT}$ & 264 & 0.994 \\
& & $\mathrm{TC}$ & 429 & \\
SCL30A8 (rs13266634) & $\mathrm{C} / \mathrm{T}$ & $\mathrm{CC}$ & 177 & \\
& & $\mathrm{CC}$ & 341 & 0.673 \\
& & $\mathrm{CT}$ & 355 & \\
TCF7L2 (rs7903146) & $\mathrm{C} / \mathrm{T}$ & $\mathrm{TT}$ & 174 & \\
& & $\mathrm{CC}$ & 833 & 0.650 \\
& & $\mathrm{CT}$ & 36 & \\
PPARG (rs1801282) & $\mathrm{C} / \mathrm{G}$ & $\mathrm{TT}$ & 1 & \\
& & $\mathrm{CC}$ & 790 & 0.796 \\
& & $\mathrm{CG}$ & 79 & \\
& & $\mathrm{GG}$ & 1 &
\end{tabular}

Abbreviations: HWE, Hardy-Weinberg equilibrium; SNPs, single nucleotide polymorphisms; T2DM, type 2 diabetes mellitus.

The Hardy-Weinberg equilibrium was analyzed by the chi-square test. Risk alleles are shown in bold type.

Table 3 Association of candidate SNPs with progression of T2DM at year 4

\begin{tabular}{lcccccc}
\hline & \multicolumn{3}{c}{ Dominant } & & \multicolumn{2}{c}{ Recessive } \\
\cline { 2 - 3 } Gene (rs number) & OR $(95 \%$ Cl) & $P$ & & OR $(95 \%$ Cl) & P \\
\hline KCNQ1 (rs2237892) & $1.27(0.70,2.28)$ & 0.43 & $2.61(1.01,6.69)$ & 0.04 \\
CDKAL1 (rs7554840) & $0.95(0.49,1.84)$ & 0.88 & $0.93(0.50,1.70)$ & 0.80 \\
CDKN2A/B (rs1081161) & $0.57(0.30,1.08)$ & 0.08 & & $1.52(0.85,2.72)$ & 0.16 \\
SCL30A8 (rs13266634) & $1.04(0.51,2.12)$ & 0.27 & $1.04(0.51,2.12)$ & 0.91 \\
TCF7L2 (rs7903146) & $0.44(0.05,3.95)$ & 0.47 & - & - \\
PPARG (rs1801282) & $0.40(0.18,0.91)$ & 0.09 & - & - \\
\hline
\end{tabular}

Abbreviations: SNPs, single nucleotide polymorphisms; T2DM, type 2 diabetes mellitus. Multivariate logistic analysis for risk factors, including genotype, age, sex, change in BMI, and baseline levels of FBG, triglyceride, HDL-C and insulin.

genotype in the recessive model (odds ratio $=2.61,95 \%$ confidence intervals $=1.02-6.69, P=0.04$; Table 3 ). No association was observed in the other SNPs. At year 4 in the C-allele carriers, after adjusting for age, sex and baseline body mass index levels, the FPG levels were significantly increased (mean value (s.e.): 5.59 (0.03) for CC+CT versus $5.50(0.08)$ for TT, $P<0.01)$ and the HOMA- $\beta$ levels were significantly decreased (108.29 (6.35) for CC+CT versus 124.54 (15.66) for TT, $P=0.03$ ) (Figure 1), although no differences were observed in baseline levels among KCNQ1 variants.

\section{DISCUSSION}

Recent genome-wide association studies of T2DM have identified several novel SNPs in type 2 diabetes susceptibility genes. ${ }^{4,9-11}$ These genetic variants have been repetitively confirmed by multiple studies in various population samples. In this study, we performed association analyses between these loci and the development of T2DM in the nondiabetic population in Korea.
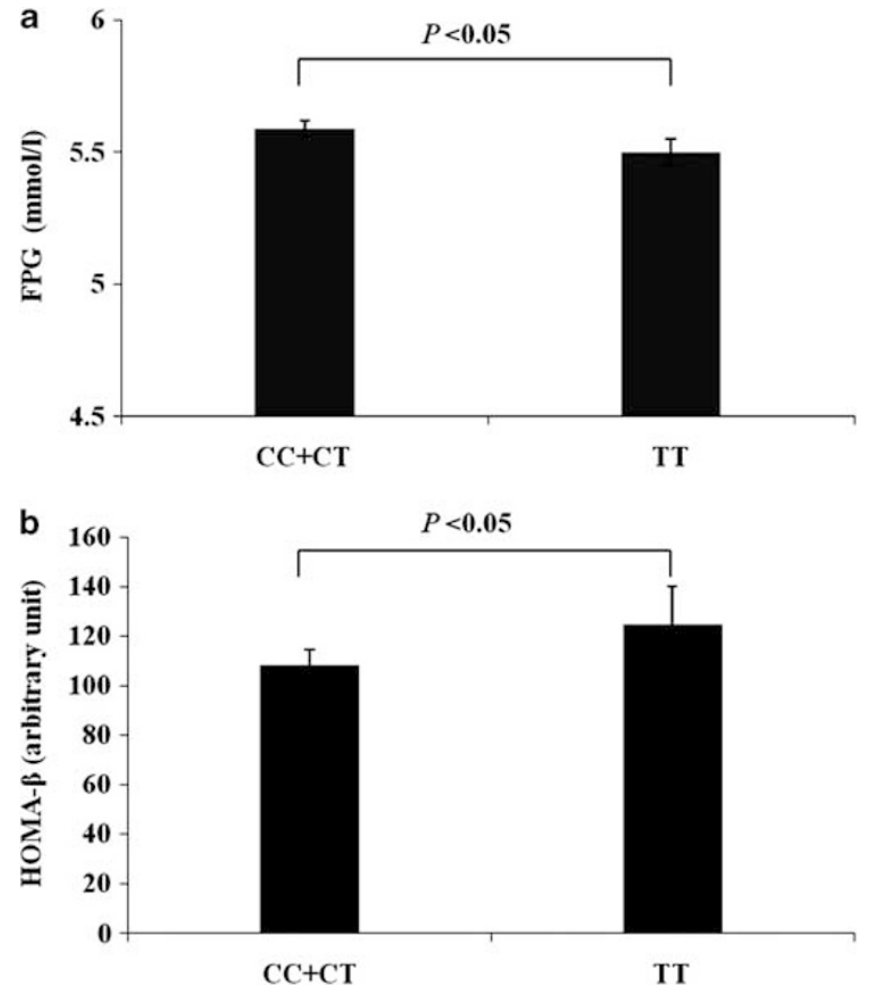

Figure 1 Comparison of KCNQ1 rs2237892 with (a) FPG and (b). HOMA- $\beta$ levels at year 4 . Data are shown as means \pm s.e. after adjustment for age, sex and body mass index.

Our present study revealed that C-allele carriers of rs2237892 in KCNQ1 had a significantly higher risk of future T2DM in a Korean population. In previous studies conducted in East Asian populations, the variant rs2237892 from intron 15 of the KCNQ1 gene was the SNP most frequently associated with T2DM and altered fasting insulin levels. ${ }^{11,24}$ Additionally, several studies reported that the KCNQ1 gene was related to diabetes in the Korean population. ${ }^{19-21}$ Most of these studies used a case subject-control subject design that tended to overestimate the risk of an SNP because case subjects and control subjects usually represented two extremes of the distribution of glucose tolerance. Therefore, we investigated whether the SNPs may indeed increase the risk of future T2DM in non-diabetic subjects. A recent study also reported that a variant in KCNQ1 rs22378985 was associated with progression to T2DM in Scandinavians. ${ }^{13}$ Our study, however, expanded previous findings by investigating the effects of various diabetogenic genes on the development of T2DM in the nondiabetic population in Korea and comparing them with longitudinal data, which distinguishes our study from others.

Thus, after 4 years, we also found significantly lower insulin secretory function (as reflected by HOMA- $\beta$ ) with the presence of the C-allele of KCNQ1, a risk allele for T2DM, although no significant differences were found at baseline among the KCNQ1 genotypes. KCNQ1 is located on $11 \mathrm{p} 15.5$ and encodes the pore-forming $\alpha$-subunit of the $\mathrm{I}_{\mathrm{KS}} \mathrm{K}^{+}$channel, which is expressed in pancreatic $\beta$ cells as well as in other tissues, such as heart and skeletal muscle. In the pancreas, KCNQ1 is co-expressed with products of other regulators, such as KCNE1, which may alter both its biophysical characteristics and its role. ${ }^{25}$ As such, it is plausible that polymorphisms within the KCNQ1 gene alter the properties and role of the $\mathrm{I}_{\mathrm{KS}} \mathrm{K}^{+}$channel, causing decreased pancreatic $\beta$ cell function and insulin production, 
eventually leading to hyperglycemia. Although the function of KCNQ1 in the pancreatic $\beta$ cell is not fully understood, several discrepancies have been noted about the relationship between the KCNQ1 variants and pancreatic $\beta$ cell function. Unoki et al. ${ }^{10}$ did not report any association with either $\beta$ cell function or insulin resistance whereas others found a significant association between the KCNQ1 variants and $\beta$ cell function. ${ }^{11,13,24}$ It was also reported that an inhibitor of KCNQ1 (that is, chromanol 293B) significantly increased insulin secretion in the pancreatic $\beta$ cell line. ${ }^{26}$ The mechanism remains unclear, but our results suggest that KCNQ1 polymorphisms may be associated with impaired insulin secretion and the development of T2DM. Further studies are needed to determine whether the KCNQ1 gene has a role in insulin secretion in humans.

In our study, no other SNPs or haplotypes were significantly associated with the progression of T2DM. Previously, several reports showed that the common genetic variants analyzed in our study were significantly associated with diabetes in the Korean population. ${ }^{19-21}$ Previous studies suggested that common variants in CDKAL1, SCL3A08, TCF7L2 and PPARG were also significantly associated with increased risk of future diabetes. ${ }^{12,27}$ These discrepancies may be explained by the differences in the frequency and loci of genetic variants, ethnicity, population characteristics and follow-up durations.

Several limitations were present in our study. First, we did not routinely perform the oral glucose tolerance test, which might lead to an underestimation of diabetes. Second, these results were obtained from relatively old people living in a rural area, which limits any extension of the results to the general Korean population. As we did not collect detailed data on the use of antihyperglycemic agents, their effects were not considered, although they can affect glucose and insulin levels. Thus, the data are subject to potential under- or overestimation. However, a few participants who took anti-diabetic drugs were included $(3.1 \%)$ without statistical significance (data not shown). Another limitation was the relatively small sample size that led to a relatively low statistical power and precluded the study of gene-gene interactions. As the value of genetic factors increased with the increasing duration of follow-up, ${ }^{27}$ longer-term follow-up of a larger number of subjects is necessary to demonstrate whether these genetic variants can lead to the development of T2DM.

In conclusion, these longitudinal data provide important evidence that genetic variations in the KCNQ1 gene are associated with future T2DM in the non-diabetic population in Korea. These findings are likely to be caused by the difference in insulin secretory function after 4 years. Further studies are warranted to elucidate the molecular mechanisms of KCNQ1 polymorphisms, as well as to evaluate their role in the development of T2DM and of impaired pancreatic $\beta$ cell function.

\section{ACKNOWLEDGEMENTS}

This work was supported by the Samsung Biomedical Research Institute Grant \#SBRI C-B0-226-1, C-B0-226-2.

1 Stumvoll, M., Goldstein, B. J. \& van Haeften, T. W. Type 2 diabetes: principles of pathogenesis and therapy. Lancet 365, 1333-1346 (2005).

2 Wild, S., Roglic, G., Green, A., Sicree, R. \& King, H. Global prevalence of diabetes: estimates for the year 2000 and projections for 2030. Diabetes Care 27, 1047-1053 (2004).
3 Singh, R. B., Bajaj, S., Niaz, M. A., Rastogi, S. S. \& Moshiri, M. Prevalence of type 2 diabetes mellitus and risk of hypertension and coronary artery disease in rural and urban population with low rates of obesity. Int. J. Cardiol. 66, 65-72 (1998).

4 Sladek, R., Rocheleau, G., Rung, J., Dina, C., Shen, L., Serre, D. et al. A genome-wide association study identifies novel risk loci for type 2 diabetes. Nature 445, 881-885 (2007).

5 Steinthorsdottir, V., Thorleifsson, G., Reynisdottir, I., Benediktsson, R., Jonsdottir, T., Walters, G. B. et al. A variant in CDKAL1 influences insulin response and risk of type 2 diabetes. Nat. Genet. 39, 770-775 (2007).

6 Saxena, R., Voight, B. F., Lyssenko, V., Burtt, N. P., de Bakker, P. I., Chen, H. et al. Genome-wide association analysis identifies loci for type 2 diabetes and triglyceride levels. Science 316, 1331-1336 (2007).

7 Scott, L. J., Mohlke, K. L., Bonnycastle, L. L., Willer, C. J., Li, Y., Duren, W. L. et al. A genome-wide association study of type 2 diabetes in Finns detects multiple susceptibility variants. Science 316, 1341-1345 (2007).

8 Zeggini, E., Scott, L. J., Saxena, R., Voight, B. F., Marchini, J. L., Hu, T. et al. Metaanalysis of genome-wide association data and large-scale replication identifies additional susceptibility loci for type 2 diabetes. Nat. Genet. 40, 638-645 (2008).

9 Zeggini, E., Weedon, M. N., Lindgren, C. M., Frayling, T. M., Elliott, K. S., Lango, H. et al. Replication of genome-wide association signals in UK samples reveals risk loci for type 2 diabetes. Science 316, 1336-1341 (2007).

10 Unoki, H., Takahashi, A., Kawaguchi, T., Hara, K., Horikoshi, M., Andersen, G. et al. SNPs in KCNQ1 are associated with susceptibility to type 2 diabetes in East Asian and European populations. Nat. Genet. 40, 1098-1102 (2008).

11 Yasuda, K., Miyake, K., Horikawa, Y., Hara, K., Osawa, H., Furuta, H. et al. Variants in KCNQ1 are associated with susceptibility to type 2 diabetes mellitus. Nat. Genet. 40, 1092-1097 (2008).

12 Florez, J. C., Jablonski, K. A., Bayley, N., Pollin, T. I., de Bakker, P. I., Shuldiner, A. R. et al. TCF7L2 polymorphisms and progression to diabetes in the Diabetes Prevention Program. N. Engl. J. Med. 355, 241-250 (2006).

13 Jonsson, A., Isomaa, B., Tuomi, T., Taneera, J., Salehi, A., Nilsson, P. et al. A variant in the KCNQ1 gene predicts future type 2 diabetes and mediates impaired insulin secretion. Diabetes 58, 2409-2413 (2009).

14 Omori, S., Tanaka, Y., Takahashi, A., Hirose, H., Kashiwagi, A., Kaku, K. et al. Association of CDKAL1, IGF2BP2, CDKN2A/B, HHEX, SLC30A8, and KCNJ11 with susceptibility to type 2 diabetes in a Japanese population. Diabetes 57, 791-795 (2008).

15 Hayashi, T., Iwamoto, Y., Kaku, K., Hirose, H. \& Maeda, S. Replication study for the association of TCF7L2 with susceptibility to type 2 diabetes in a Japanese population. Diabetologia 50, 980-984 (2007).

16 Kwon, H. S., Park, Y. M., Lee, H. J., Lee, J. H., Choi, Y. H., Ko, S. H. et al. Prevalence and clinical characteristics of the metabolic syndrome in middle-aged Korean adults. Korean J. Intern. Med. 20, 310-316 (2005).

17 Diagnosis and classification of diabetes mellitus. Diabetes Care $34($ Suppl 1), S62-S69.

18 Matthews, D. R., Hosker, J. P., Rudenski, A. S., Naylor, B. A., Treacher, D. F. \& Turner, R. C. Homeostasis model assessment: insulin resistance and beta-cell function from fasting plasma glucose and insulin concentrations in man. Diabetologia 28, 412-419 (1985).

19 Cho, Y. M., Kim, T. H., Lim, S., Choi, S. H., Shin, H. D., Lee, H. K. et al. Type 2 diabetes-associated genetic variants discovered in the recent genome-wide association studies are related to gestational diabetes mellitus in the Korean population. Diabetologia 52, 253-261 (2009).

20 Kang, E. S., Kim, M. S., Kim, C. H., Nam, C. M., Han, S. J., Hur, K. Y. et al. Association of common type 2 diabetes risk gene variants and posttransplantation diabetes mellitus in renal allograft recipients in Korea. Transplantation 88, 693-698 (2009).

21 Lee, Y. H., Kang, E. S., Kim, S. H., Han, S. J., Kim, C. H., Kim, H. J. et al. Association between polymorphisms in SLC30A8, HHEX, CDKN2A/B, IGF2BP2, FTO, WFS1, CDKAL1, KCNQ1 and type 2 diabetes in the Korean population. J. Hum. Genet. 53, 991-998 (2008).

22 Ng, M. C., Park, K. S., Oh, B., Tam, C. H., Cho, Y. M., Shin, H. D. et al. Implication of genetic variants near TCF7L2, SLC30A8, HHEX, CDKAL1, CDKN2A/B, IGF2BP2, and FTO in type 2 diabetes and obesity in 6719 Asians. Diabetes 57, 2226-2233 (2008).

23 Horikawa, Y., Miyake, K., Yasuda, K., Enya, M., Hirota, Y., Yamagata, K. et al. Replication of genome-wide association studies of type 2 diabetes susceptibility in Japan. J. Clin. Endocrinol. Metabol. 93, 3136-3141 (2008).

24 Tan, J. T., Nurbaya, S., Gardner, D., Ye, S., Tai, E. S. \& Ng, D. P. Genetic variation in KCNQ1 associates with fasting glucose and beta-cell function: a study of 3734 subjects comprising three ethnicities living in Singapore. Diabetes 58, 1445-1449 (2009).

25 Warth, R., Garcia Alzamora, M., Kim, J. K., Zdebik, A., Nitschke, R., Bleich, M. et al. The role of KCNQ1/KCNE1 K(+) channels in intestine and pancreas: lessons from the KCNE1 knockout mouse. Pflugers. Arch. 443, 822-828 (2002).

26 Ulrich, S., Wolter, F. \& Stein, J. M. Molecular mechanisms of the chemopreventive effects of resveratrol and its analogs in carcinogenesis. Mol. Nutr. Food. Res. 49, 452-461 (2005).

27 Lyssenko, V., Jonsson, A., Almgren, P., Pulizzi, N., Isomaa, B., Tuomi, T. et al. Clinical risk factors, DNA variants, and the development of type 2 diabetes. N. Engl. J. Med. 359, 2220-2232 (2008) 